\title{
Chaotic Ant Swarm Based Parameter Estimation of Induction Motor from Manufacturer Data
}

\author{
Li Yan-jie $^{\text {a }}$, Jia Zhu-zhi ${ }^{\text {b }}$ \\ School of Higher Vocational and Technology, University of Science and Technology Liaoning, \\ China \\ a li_yanjie@126.com, bjiazhuzhi@163.com
}

Keywords: Chaotic Ant Swarm Algorithm, Induction Motor, Parameter Estimation

\begin{abstract}
A method of parameter estimation of induction motor based on optimization using a chaotic swarm algorithm is presented. The parameter estimation methodology describes a method for estimating the steady-state equivalent circuit parameters from the motor performance characteristic, which is normally available from manufacturer data or from tests. The optimization problem is formulated as multi-objective function to minimize the error between the estimated and the manufacturer data. Chaotic ant swarm algorithm is a novel optimization method, which has the ability of global optimum search. A numerical simulation on the test motor is conducted. Simulation results show that the proposed method is effective in parameter estimation of the induction motor.
\end{abstract}

\section{Introduction}

Induction machine models used for the solution of a variety of steady-state problems require equivalent circuit parameters. These parameters include the resistances and reactances representing the stator, rotor and magnetizing branches. The main problem of induction motor parameter estimation is the unavailability of manufacturer data to construct accurate models. Due to this reason, the induction motor models are not explicitly represented in various applications.

The conventional technique for estimating the induction motor parameters are based on the no-load and the locked-rotor tests[1]. However, these approaches cannot be implemented easily. Besides, the locked-rotor test requires that the shaft of the motor be locked. In the locked-rotor condition, the frequency of the rotor is equal to the supply frequency, but under typical operations, the rotor frequency is perhaps $1-3 \mathrm{~Hz}$. This incorrect rotor frequency will give misleading results for the locked-rotor test. In the recent years, global optimization techniques such as evolutionary algorithm[2], genetic algorithm[3,4], adaptive genetic algorithm[5] and differential evolution[6] have been proposed to solve the parameter estimation problems.

Though the genetic algorithm methods have been employed successfully to solve complex non-linear optimization problems, recent research has identified some deficiencies in GA[7]. This degradation in efficiency is apparent when the parameters being optimized are highly correlated and the premature convergence of the GA degrades its performance in terms of reducing the search capability of genetic algorithm(GA).

Chaotic ant swarm algorithm was inspired by behaviors of real ants of nature. This novel method includes both effects of chaotic dynamics and swarm-based search. It is a deterministic process different from the conventional ant algorithm. Chaotic ant swarm algorithm has the ability of global optimum search and can generate high-quality solutions.

\section{Chaotic Ant Swarm Algorithm}

Algorithms inspired by models of ant colony behaviors have achieved increasing success among researchers in computer science and operations research. Much of the knowing developed ant-inspired optimization algorithms are based on the random metaheuristic of non-deterministic probability theory. However, it has been discovered by biologists that single ant shows chaotic 
activity patterns. And from the viewpoint of kinetics, some certain inherent relations between the chaotic activity patterns of single ant and the self-organizing behavior of the colony exist. The chaotic behavior of individual ant and the intelligent organization actions of ant colony are adaptations to the environment. These behaviors are very important for the ant to find food and survive[8,9]. Inspired by natural ants behavior, Reference [10,11] combined the ant chaotic kinetics, swarm organizing, and optimization mechanism ingeniously, and proposed a new kind of optimization algorithm based on swarm intelligent theory, which is chaotic ant swarm algorithm. The mathematic model of the chaotic ant swarm is as follows:

$$
\left\{\begin{array}{l}
\mu_{i}(n)=\mu_{i}(n-1)^{\left(1+r_{i}\right)} \\
\theta_{i d}(n)=\left[\theta_{i d}(n-1)+\frac{7.5}{\psi_{i d}} \times V_{i}\right] \times \exp \left\{[ 1 - \operatorname { e x p } ( - \alpha \mu _ { i } ( n ) ) ] \left[3-\psi_{i d}\left(\theta_{i d}(n-1)+\right.\right.\right. \\
\left.\left.\left.\frac{7.5}{\psi_{i d}} \times V_{i}\right)\right]\right\}-\frac{7.5}{\psi_{i d}} \times V_{i}+\exp \left[-2 \alpha \mu_{i}(n)+\delta\right]\left[P_{i d}(n-1)-\theta_{i d}(n-1)\right]
\end{array}\right.
$$

where $\mu_{i}$ is the organization variable of an ant swarm system, and $\mu_{i}(0)=0.999 ; r_{i}$ is organization factor; $\theta_{i d}$ is the $d$ dimension state of $i$ th ant, $d=1,2, \cdots, L, L$ is the dimension of optimization space, $I$ $=1,2, \cdots, N, N$ is the number of the ants; $P_{i d}(n-1)$ is the best position found by $i$ th ant and its neighbors within $n-1$ steps; $\psi_{d}$ is a constant and can be used to adjust the search range of $\boldsymbol{\theta}_{i d} ; V_{i}$ determines the moving proportion of the ant chaotic kinetic behavior equation in the space, and generally $V_{i}=1 / 2 ; \delta$ is a constant and $0 \leq \delta \leq 2 / 3 ; \alpha$ is a very large positive constant, such as $\alpha=200[11]$.

We suppose there are two ants whose positions are $\left(\theta_{i 1}, \cdots, \theta_{i L}\right)$ and $\left(\theta_{j 1}, \cdots, \theta_{j L}\right)$ respectively, where $i \neq j, i, j=1, \cdots, N$ and $i \neq j$, then the distance between the two ants is

$$
\sqrt{\left(\theta_{i 1}-\theta_{j 1}\right)^{2}+\ldots+\left(\theta_{i L}-\theta_{j L}\right)^{2}}
$$

We define the nearest $M$ ants in distance as the neighbors of single ant $i$, and $M<N$. The optimization information $P_{i d}$ is conveyed among the single ant $i$ and its neighbors. Obviously, when the number of ants $N$ is even, and if $M \geq N / 2$, the ants will converge at the same optimum or near-optimum; and when $N$ is odd, if $M \geq(N-1) / 2$, the ants will converge at the same optimum or near-optimum. At other circumstances, not all the ants can converge at the same value, that is to say, some of the ants can't find the optimum or near-optimum[11]. $r_{i}$ and $\psi_{d}$ are two important parameters in (1). $r_{i}$ has effect on the convergence speed of chaotic ant swarm algorithm. If the organization factor $r_{i}$ is very large, the system converges quickly and the chaos state of chaotic ant swarm disappears, thus we can't achieve the desired optima or near-optima. If the organization factor $r_{i}$ is very small, the system converges slowly and the runtime will be longer. If $r_{i}$ equals to zero, the system of chaotic ant swarm is chaotic and won't converge, thus it can't achieve the objective of optimization. Further, since small changes are desired as time evolves, the value of $r_{i}$ is chosen typically $0<r_{i} \leq 0.5$. The format of $r_{i}$ can be designed according to concrete problems and runtime. In order to denote that each ant has different $r_{i}$, for example, we can set it as $r_{i}=0.1+$ $0.2 \times \operatorname{rand}()$, where $\operatorname{rand}()$ is a uniformly distributed random number in $[0,1] . \psi_{d}>0$ has effect on the searching ranges of the proposed method. If the parameter $\psi_{d}$ is very small, the searching ranges of the algorithm is large; and if it is large, the searching ranges is small. We assume the search arrange is $\left[-\omega_{d} / 2, \omega_{d} / 2\right]$, then $\omega_{d} \approx 7.5 / \psi_{d}$.

\section{Parameter Estimation of Induction Motor}

\section{Approximate Circuit Model Formulation.}

An induction motor can be modeled by using an approximate model, an exact model or a deep bar model[1]. The parameter estimation problem is formulated as a least squares optimization problem, the objective being the minimization of deviation between the estimated and the manufacturer data. In this paper, we adopt the exact circuit model to estimate the parameters. The problem formulation for the parameter estimation of the exact circuit model is described below.

The problem formulation uses the starting torque, the maximum torque and the full load torque 
manufacturer data to estimate the stator resistance, the rotor resistance and the magnetizing reactance parameter $X_{m}$ is not considered in this model. The exact circuit model of the induction motor is shown in Figure1. The objective function and associated constraints of the problem are formulated as follows:

Objective function $\quad F_{\min }=f_{1}^{2}+f_{2}^{2}+f_{3}^{2}+f_{4}^{2}$,

Where $f_{1}=\frac{\frac{K_{t} R_{2}}{s\left[\left(R_{t h}+\frac{R_{2}}{s}\right)^{2}+X^{2}\right]}-T_{f l}(m)}{T_{f l}(m)}, \quad f_{2}=\frac{\frac{K_{t} R_{2}}{\left(R_{t h}+R_{2}\right)^{2}+X^{2}}-T_{l r}(m)}{T_{l r}(m)}, \quad f_{3}=\frac{\frac{K_{t}}{2\left(R_{t h}+\sqrt{R_{t h}{ }^{2}+X^{2}}\right)}-T_{\max }(m)}{T_{\max }(m)}$,

$f_{4}=\frac{\cos \left(\tan ^{-1}\left(\frac{X}{R_{t h}+\frac{R_{2}}{s}}\right)\right)-p f_{f l}(m)}{p f_{f}(m)}, \quad V_{t h}=\frac{V_{p h} X_{m}}{X_{1}+X_{m}}, \quad R_{t h}=\frac{R_{1} X_{m}}{X_{1}+X_{m}}, \quad X_{t h}=\frac{X_{1} X_{m}}{X_{1}+X_{m}}, \quad K_{t}=\frac{3 V_{t h}^{2}}{\omega_{s}}, X=X_{2}+X_{\mathrm{th}}$.

$V_{p h}$ is stator voltage per phase; $\omega_{\mathrm{s}}$ is motor angular velocity; $R_{1}$ is the stator resistance; $R_{2} / s$ is rotor resistance referred to stator side; $X_{1}$ is stator leakage reactance per phase; $X_{2}$ is rotor reactance referred to stator side; $V_{\mathrm{th}}, R_{\mathrm{th}}, X_{\mathrm{th}}$ are thevenin's equivalent voltage, resistance, and reactance, respectively; where ' $m$ ' stands for the manufacturer. $T_{f l}(m), T_{l r}(m), T_{\max }(m)$ and $p f_{f l}(m)$ are the manufacturer values of the full load torque, the locked-rotor torque, the maximum torque and full load power factor, respectively.

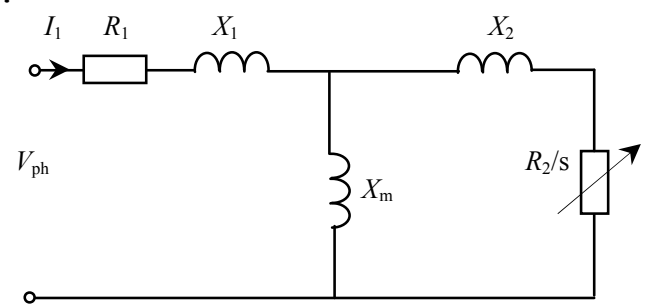

Figure1. Exact circuit model of induction motor

\section{Constraints.}

Based on the nameplate data, the following constraints are defined[12]:

$$
X_{1}=X_{2}, \frac{T_{\max }-T_{\max }(m)}{T_{\max }(m)} \leq \pm 0.2
$$

where $T_{\max }$ is the estimated maximum torque.

\section{Parameter Estimation of Induction Motor.}

The principles of the chaotic ant swarm algorithm are described in section 2. Its procedure for parameter estimation can be summarized as follows:

Step 1: Get the manufacturer data of the induction motor.

Step2: Generate initial positions of all ants at random in the searching space, and $\theta_{i}^{0}=\left(\theta_{i 1}^{0}, \ldots, \theta_{i L}^{0}\right)^{T}$ is the initial position of $i$ th ant, subscript $\mathrm{i}=1,2, \ldots, N$, where $N$ is the number of ants, $L$ is the dimension of optimization space, superscript 0 is the initial state. The searching range is decided by $\psi_{d}$, where $d$ is the $d$ th elementary in the searching space.

Step3: Define the object function as equation (3), and the problem of parameter estimation of the induction motor is converted to a problem of parameter $\boldsymbol{\theta}$ optimization which can make the object function minimum.

Step4: Put $\hat{\theta}_{i}^{n}$ into (3) in every iterative step, and compute the corresponding object function.

Step5: The estimation process ends, and output corresponding results, if the object function values of all ants meet the ending condition.

\section{Simulation Results}

In order to evaluate the effectiveness of the chaotic ant swarm algorithm, we apply the chaotic ant swarm algorithm on the system parameters recognition problem, and compare the results of the proposed method with that of GA method. The simulation condition is Intel Core 2 Duo processor T6600, 2.20GHz, WindowsXP, Matlab.R2011a. The manufacturer data of the test motor is shown as 
Table 1.

Table 1 Manufacturer Data of The Test Motor

\begin{tabular}{cccccc}
\hline Specifications & Motor & Specifications & Motor & Specifications & Motor \\
\hline Capability(HP) & 5 & No. of Poles & 4 & Maximum torque(Nm) & 42 \\
Voltage(V) & 400 & Full load power factor & 0.8 & Starting current(A) & 22 \\
Current(A) & 8 & Full load slip & 0.07 & Full load torque(Nm) & 25 \\
Frequency(Hz) & 50 & Starting torque(Nm) & 15 & & \\
\hline
\end{tabular}

In the process of computer simulation, we select 20 as the number of individual ants, and adopt global neighbors model, that is the number of ant neighbors is $M=N-1$. Let $\mu(0)=0.999, \alpha=200$, $r_{i}=0.1+0.2 \mathrm{rand}()$, where rand() is random number in $[0,1]$. Let $\delta=2 / 3, V_{i}=0, \psi_{1}=0.75, \psi_{2}=0.75$, $\psi_{3}=0.15, \psi_{4}=0.015$, so the searching range of parameter $R_{1}$ is $[0,10]$, the searching range of parameter $R_{2}$ is $[0,10]$, the searching range of parameters $X_{1}$ and $X_{2}$ are [0,50], and the searching range of parameter $X_{\mathrm{m}}$ is $[0,500]$. And we run the chaotic ant swarm algorithm to estimate the 5 unknown parameters. The results of the proposed method and GA method are shown in Table 2-5. Figure2-Figure4 shows the results of the chaotic ant swarm algorithm. From these figures, we can see that the accuracy of the chaotic ant swarm is higher than that of GA method. The CAS results of $T_{\mathrm{st}}, T_{\max }$ and $T_{\text {ful }}$ are far better than those of GA. So CAS parameter estimation method has a higher quality than GA method as a whole.

Table2 The Results of GA Eatimating Model Parameters

\begin{tabular}{ccccc}
\hline Parameters & \multicolumn{4}{c}{$\mathrm{GA}$} \\
\hline$R_{1}(\Omega)$ & 2.3247 & 2.4167 & 2.5827 & 2.4782 \\
$R_{2}(\Omega)$ & 7.4592 & 7.2048 & 7.3307 & 7.0275 \\
$X_{1}, X_{2}(\Omega)$ & 15.8732 & 15.9481 & 16.4612 & 16.7729 \\
$X_{\mathrm{m}}(\Omega)$ & 132.437 & 130.662 & 135.254 & 128.9926 \\
\hline
\end{tabular}

Table 3 The Results of CAS Eatimating Model Parameters

\begin{tabular}{ccccc}
\hline Parameters & \multicolumn{4}{c}{ CAS } \\
\hline$R_{1}(\Omega)$ & 1.8916 & 1.9011 & 1.8825 & 1.9476 \\
$R_{2}(\Omega)$ & 5.9037 & 5.8927 & 5.9157 & 5.9211 \\
$X_{1}, X_{2}(\Omega)$ & 15.4118 & 15.4610 & 15.3290 & 15.4525 \\
$X_{\mathrm{m}}(\Omega)$ & 150.429 & 151.004 & 148.981 & 150.4479 \\
\hline
\end{tabular}

Table4 GA Estimating Results

\begin{tabular}{ccccccccc}
\hline $\begin{array}{c}\text { Torque } \\
(\mathrm{Nm})\end{array}$ & $\begin{array}{c}\text { Estimated } \\
\text { data }\end{array}$ & $\begin{array}{c}\text { Error } \\
(\%)\end{array}$ & $\begin{array}{c}\text { Estimate } \\
\text { d data }\end{array}$ & $\begin{array}{c}\text { Error } \\
(\%)\end{array}$ & $\begin{array}{c}\text { Estimat } \\
\text { ed data }\end{array}$ & $\begin{array}{c}\text { Error } \\
(\%)\end{array}$ & $\begin{array}{c}\text { Estimat } \\
\text { ed data }\end{array}$ & $\begin{array}{c}\text { Error } \\
(\%)\end{array}$ \\
\hline$T_{s t}$ & 18.2896 & 21.93 & 17.5344 & 16.90 & 16.7600 & -13.76 & 15.5054 & 0.0337 \\
$T_{\max }$ & 37.8424 & -9.90 & 37.4649 & -10.80 & 36.2226 & 11.73 & 35.3107 & -0.1593 \\
$T_{\text {ful }}$ & 20.4376 & -18.25 & 20.9024 & -16.39 & 20.4712 & -18.12 & 20.8479 & -0.1661 \\
\hline
\end{tabular}

Table5 CAS Estimating Results

\begin{tabular}{ccccccccc}
\hline $\begin{array}{c}\text { Torque } \\
(\mathrm{Nm})\end{array}$ & $\begin{array}{c}\text { Estimated } \\
\text { data }\end{array}$ & $\begin{array}{c}\text { Error } \\
(\%)\end{array}$ & $\begin{array}{c}\text { Estimate } \\
\text { d data }\end{array}$ & $\begin{array}{c}\text { Error } \\
(\%)\end{array}$ & $\begin{array}{c}\text { Estimat } \\
\text { ed data }\end{array}$ & $\begin{array}{c}\text { Error } \\
(\%)\end{array}$ & $\begin{array}{c}\text { Estimate } \\
\text { d data }\end{array}$ & $\begin{array}{c}\text { Error } \\
(\%)\end{array}$ \\
\hline$T_{s t}$ & 16.1004 & 7.34 & 15.9764 & 6.51 & 16.2888 & 8.59 & 16.0461 & 6.97 \\
$T_{\max }$ & 40.3472 & -3.94 & 40.2180 & -4.24 & 40.5396 & -3.48 & 40.1639 & -4.37 \\
$T_{f u l}$ & 25.6427 & 2.57 & 25.6598 & 2.64 & 25.6173 & 2.47 & 25.5336 & 2.13 \\
\hline
\end{tabular}

\section{Conclusions}

This paper proposes a new parameter estimation method for induction motor based on chaotic ant swarm algorithm. The problem of induction motor parameter estimation is converted to a problem of parameter optimization which could be solved via chaotic ant swarm algorithm. Chaotic 
ant swarm algorithm has the ability of global optimum search. A numerical simulation on the test motor is conducted. Simulation results show that CAS parameter estimation method has a higher quality than GA method. So the proposed method is effective in parameter estimation of the induction motor.

An induction motor can be modeled by using an approximate circuit model, an exact circuit model or a deep bar circuit model. In this paper, we adopt the exact circuit model, and next we will research the deep bar circuit model to estimate parameters of induction motors.

\section{References}

[1] V.P.Sakthivel, R.Bhuvaneswari, S.Subramanian, Multi-objective Parameter Estimation of Induction Motor Using Particle Swarm Optimization. Engineering Applications of Artificial Intelligence. 2010; Vol.23: 302-312

[2] Nangsue P, Pillay P, Conry S. Evolutionary Algorithms for Induction Motor Parameter Determination. IEEE Transactions Energy Conversion.1999; Vol.14:447-453

[3] Orlowska Kowalska, Lis J, Szabat K. Identification of The Induction Motor Parameters Using Soft Computing Methods. Computation and Mathematics in Electrical and Electronics Engineering. 2006; Vol.25:181-192

[4] Rahimpour E, Rashtchi V, Pesaran M. Parameter Identification of Deep-bar Induction Motors Using Genetic Algorithm. Electrical Engineering.2007;Vol.89:547-552

[5] Abdelhadi B, Benoudjit A, Nait Said. Identification of Induction Machine Parameters Using an Adaptive Genetic Alogrithm. Electric Power Components And Systems.2004; Vol.32:767-784

[6] Ursem RK,Vadstrup P. Parameter Identification of Induction Motors Using Differential Evolution. The 2003 Congress on Evolutionary Computation, Canberra, 2003:790-796

[7] Fogel DB, Evolutionary Computation: Toward a New Philosophy of Machine Intelligence, second IEEE Press, Piscataway,NJ.2000

[8] Li Li-xiang, Peng Hai-peng, Wang Xiang-dong, et al. PID parameter tuning based on chaotic ant swarm, Chinese Journal of Scientific Instrument, 2006; Vol.27:1104-1106.

[9] Li Li-xiang, Yang Yi-xian, Peng Hai-peng, et al. Parameters Identification of Chaotic Systems Via Chaotic Ant Swarm. Chaos, Solitons \& Fractals, 2006; Vol.28:1204-1211

[10] Li LX, Peng Hai-peng, Wang Xiang-dong, et al. An optimization method inspired by " chaotic" ant behavior. International Journal of Bifurcation and Chaos,2006; Vol.16: 2351-2364

[11] Li Li-xiang, Peng Hai-peng, Yang Yi-xian, et al. Parameter estimation for Lorenz Chaotic systems based on chaotic ant swarm algorithm, Acta Phys, 2007; Vol.56:51-55.

[12] V.P.Sakthivel, R.Bhuvaneswari, S.Subramanian, Bacterial foraging Technique Based Parameter Estimation of Induction Motor from Manufacturer Data, Electric Power Components and Systems, 2010; Vol38: 657-674 